\title{
A Pseudo-Boolean Framework for Computing Rearrangement Distances between Genomes with Duplicates
}

\author{
Sébastien Angibaud* Guillaume Fertin ${ }^{\dagger} \quad$ Irena Rusu ${ }^{\ddagger}$ \\ Stéphane Vialette ${ }^{\S}$
}

22nd February 2007

\begin{abstract}
Computing genomic distances between whole genomes is a fundamental problem in comparative genomics. Recent researches have resulted in different genomic distance definitions: number of breakpoints, number of common intervals, number of conserved intervals, Maximum Adjacency Disruption number (MAD), etc. Unfortunately, it turns out that, in presence of duplications, most problems are NP-hard, and hence several heuristics have been recently proposed. However, while it is relatively easy to compare heuristics between them, until now very little is known about the absolute accuracy of these heuristics. Therefore, there is a great need for algorithmic approaches that compute exact solutions for these genomic distances. In this paper, we present a novel generic pseudo-boolean approach for computing the exact genomic distance between
\end{abstract}

*Laboratoire d'Informatique de Nantes-Atlantique (LINA), FRE CNRS 2729 - Université de Nantes, 2 rue de la Houssinière, 44322 Nantes Cedex 3, France. E-mail: Sebastien.Angibaud@univ-nantes.fr

${ }^{\dagger}$ Laboratoire d'Informatique de Nantes-Atlantique (LINA), FRE CNRS 2729 - Université de Nantes, 2 rue de la Houssinière, 44322 Nantes Cedex 3, France. E-mail: Guillaume.Fertin@univ-nantes.fr

†Laboratoire d’Informatique de Nantes-Atlantique (LINA), FRE CNRS 2729 - Université de Nantes, 2 rue de la Houssinière, 44322 Nantes Cedex 3, France. E-mail: Irena.Rusu@univ-nantes.fr

${ }^{\S}$ Laboratoire de Recherche en Informatique (LRI), UMR CNRS 8623 - Faculté des Sciences d'Orsay Université Paris-Sud, 91405 Orsay, France. E-mail: vialette@lri.fr 
two whole genomes in presence of duplications, and put strong emphasis on common intervals under the maximum matching model. Of particular importance, we show three heuristics which provide very good results on a well-known public dataset of $\gamma$ Proteobacteria.

Keywords: pseudo-boolean programming, genome rearrangement, common intervals, duplication, heuristic.

\section{Introduction}

Due to the increasing amount of completely sequenced genomes, the comparison of gene order is now becoming a standard approach in comparative genomics. A natural way to compare species is to compare their whole genomes, where comparing two genomes is very often realized by determining a measure of similarity (or dissimilarity) between them. Several similarity (or dissimilarity) measures between two whole genomes have been proposed and studied in the past few years, such as the number of breakpoints (Sankoff, 1999; Bryant, 2000; Blin et al., 2004; Goldstein et al., 2004; Chrobak et al., 2005), the number of reversals (Bryant, 2000; El-Mabrouk, 2001; El-Mabrouk, 2002; Marron et al., 2004; Chen et al., 2005; Swenson et al., 2005a; Swenson et al., 2005b; Fu et al., 2006; Kolman and Waleń, 2006; Kolman and Waleń, 2007), the number of conserved intervals (Blin and Rizzi, 2005; Chen et al., 2006a), the number of common intervals (Bourque et al., 2005), the Maximum Adjacency Disruption Number (MAD) (Sankoff and Haque, 2005), etc. However, in the presence of duplications and for each of the above measures, one has first to disambiguate the data by inferring homologs, i.e., a non-ambiguous mapping between the genes of the two genomes. 
Up to now, two extremal approaches have been considered: the exemplar matching model and the maximum matching model. In the exemplar matching model (Sankoff, 1999), for all duplicated genes, all but one occurrence in each genome are deleted. In the maximum matching model (Blin et al., 2004; Goldstein et al., 2004; Chrobak et al., 2005; Chen et al., 2005; Fu et al., 2006; Chauve et al., 2006; Kolman and Waleń, 2007), the goal is to map as many genes as possible. These two models can be considered as the extremal cases of the same generic homolog assignment approach.

Unfortunately, it has been shown that, for each of the above mentioned measures, whatever the considered model (exemplar or maximum matching), the problem becomes NP-hard as soon as duplicates are present in genomes (Bryant, 2000; Blin et al., 2004; Blin and Rizzi, 2005; Chauve et al., 2006) ; some inapproximability results are known for some special cases (Thach, 2005; Chen et al., 2006b; Chen et al., 2006a). Therefore, several heuristic methods have been recently devised to obtain (hopefully) good solutions in a reasonable amount of time (Blin et al., 2005; Bourque et al., 2005). However, while it is relatively easy to compare heuristics between them, until now very little is known about the absolute accuracy of these heuristics. Therefore, there is a great need for algorithmic approaches that compute exact solutions for these genomic distances.

In the present paper, we introduce a novel generic pseudo-boolean programming approach for computing exact solutions. In this first attempt, we focus on the problem of finding the maximum number of common intervals between two genomes under the maximum matching model. From a computational point of view, the problem of computing a matching that maximizes the number of common intervals (together with MAD) is one of the hardest in our pseudo-boolean framework. Moreover, this allows us to present with a single example 
the main idea of our approach: a pseudo-boolean program together with reduction rules. We also present three heuristics for solving this problem, and we compare their results to the ones obtained by the pseudo-boolean program on a dataset of $\gamma$-Proteobacteria.

This paper is organized as follows. In Section 2, we present some preliminaries and definitions. We focus in Section 3 on the problem of finding the maximum number of common intervals under the maximum matching model, and give a pseudo-boolean programming approach together with some reduction rules. Section 4 is devoted to experimental results on a dataset of $\gamma$-Proteobacteria. In particular, in this section we define three different heuristics and we prove that they provide very good results on our dataset.

\section{Preliminaries}

Genomes with duplications are usually represented by signed sequences over the alphabet of gene families, where every element in a genome is a gene. However, in order to simplify notations, and since common intervals do not depend on the sign given to the genes, we will consider only unsigned genomes in the rest of the paper. Any gene belongs to a gene family, and two genes belong to the same gene family if they have the same label. In the sequel, we will be extensively concerned with pairs of genomes. Let $G_{1}$ and $G_{2}$ be two genomes, and let $a \in\{1,2\}$. The number of genes in genome $G_{a}$ is always written $n_{a}$. We denote the $i$-th gene of genome $G_{a}$ by $G_{a}[i]$. For any $1 \leq i \leq j \leq n_{a}$, we write $\mathcal{G}_{a}(i, j)$ for the set $\left\{G_{a}[i], G_{a}[i+1], \ldots, G_{a}[j]\right\}$ and we let $\mathcal{G}_{a}$ stand for $\mathcal{G}_{a}\left(1, n_{a}\right)$. In other words, $\mathcal{G}_{a}(i, j)$ is the set of all distinct genes between positions $i$ and $j$ in genome $G_{a}$, while $\mathcal{G}_{a}$ is the set of all distinct genes in the whole genome $G_{a}$. For any gene $\mathbf{g} \in \mathcal{G}_{a}$ and any $1 \leq i \leq j \leq n_{a}$, we denote by $\operatorname{occ}_{a}(\mathbf{g}, i, j)$ the number of occurrences of gene $\mathbf{g}$ in the sequence $\left(G_{a}[i], G_{a}[i+1], \ldots, G_{a}[j]\right)$. 
To simplify notations, we abbreviate $\operatorname{occ}_{a}\left(\mathbf{g}, 1, n_{a}\right)$ to $\operatorname{occ}_{a}(\mathbf{g})$.

A matching $\mathcal{M}$ between genomes $G_{1}$ and $G_{2}$ is a set of pairwise disjoint pairs $\left(G_{1}[i], G_{2}[j]\right)$, where $G_{1}[i]$ and $G_{2}[j]$ belong to the same gene family, i.e., $G_{1}[i]=G_{2}[j]$. Genes of $G_{1}$ and $G_{2}$ that do not belong to any pair of the matching $\mathcal{M}$ are said to be unmatched for $\mathcal{M}$. A matching $\mathcal{M}$ between $G_{1}$ and $G_{2}$ is said to be maximum if for any gene family $f$, there are no two genes of $f$ that are unmatched for $\mathcal{M}$ and belong to $G_{1}$ and $G_{2}$, respectively. A matching $\mathcal{M}$ between $G_{1}$ and $G_{2}$ is said to be exemplar if for any gene family $f$, exactly one gene of $f$ is matched by $\mathcal{M}$ between $G_{1}$ and $G_{2}$. A matching $\mathcal{M}$ between $G_{1}$ and $G_{2}$ can be seen as a way to describe a putative assignment of orthologous pairs of genes between $G_{1}$ and $G_{2}$ (see for example (Chen et al., 2005) ; see also Figure 1 for an illustration of the exemplar and maximum matching concepts).

Let $\mathcal{M}$ be any matching between $G_{1}$ and $G_{2}$. By first deleting unmatched genes and next renaming genes in $G_{1}$ and $G_{2}$ according to the matching $\mathcal{M}$, we may now assume that both $G_{1}$ and $G_{2}$ are duplication-free, i.e., $G_{2}$ is a permutation of $G_{1}$.

It is easily seen that, by first resorting to a renaming procedure, we can always assume that one of the two genomes, say $G_{1}$, is the identity permutation, i.e., $G_{1}=12 \ldots n_{1}$ (an illustration is shown in the bottom part of Figure 1).

A common interval between $G_{1}$ and $G_{2}$ is a substring of $G_{1}$, i.e., a sequence of consecutive genes of $G_{1}$, for which the exact same content can be found in a substring of $G_{2}$ (see for instance (Uno and Yagiura, 2000; Landau et al., 2005; Bergeron et al., 2005)). For example, let $G_{1}=12345$ and $G_{2}=15342$. Then, the interval [3:5] of $G_{1}$ is a common interval (because 534 occurs as a substring in $G_{2}$ ). Notice that there exists at least $n+1$ ( $n=n_{1}=n_{2}$ ) common intervals between $G_{1}$ and $G_{2}$ since each individual gene is always 
a common interval and $G_{1}$ itself is also a common interval. This lower bound is tight as shown by $G_{1}=1234$ and $G_{2}=2413$. Furthermore, if $G_{1}=G_{2}$ the number of common intervals between $G_{1}$ and $G_{2}$ is $\frac{n(n+1)}{2}$, where $n=n_{1}=n_{2}$, i.e., each possible substring of $G_{1}$ is a common interval.

-Figure 1 should go here-

\section{An exact algorithm for maximizing the number of common intervals}

In this section, we are interested in the following problem: given two genomes that contain duplicates, find a maximum matching that maximizes the number of common intervals. We show how this problem can be transformed into an equivalent pseudo-boolean problem. The section is organized as follows: in Section 3.1, we briefly introduce basic and general notions concerning pseudo-boolean programs. Section 3.2 shows how to transform our initial problem into a pseudo-boolean program, while Section 3.3 shows how this program can be simply modified in order to compute the maximum number of common intervals between two genomes under two other models: the exemplar matching model and the intermediate model, where at least one gene in each gene family is mapped. Finally, Section 3.4 contains a set of rules which will help speed-up the program by avoiding the generation of a large number of variables and constraints. 


\subsection{Pseudo-boolean models}

A Linear Pseudo-boolean (LPB) program is a linear program (Schrijver, 1998) where all variables are restricted to take values of either 0 or 1 . For one, LPB programs are viewed by the linear programming community as just a domain restriction on general linear programming. For another, from a satisfiability (SAT) point of view, pseudo-boolean constraints can be seen as a generalization of clauses providing a significant extension of purely propositional constraints (Chai and Kuehlmann, 2003; Eén and Sörensson., 2006).

Conventionally, LPB problems are handled by generic Integer Linear Programming (ILP) solvers. The drawback of such an approach is that generic ILP solvers typically ignore the boolean nature of the variables. Alternatively, LPB decision problems could be encoded as SAT instances in pure CNF (Conjunctive Normal Form), i.e., conjunction of disjunctions of boolean literals, which are then solved by any of the highly specialized SAT approaches. However the number of clauses required for expressing the LPB constraints is prohibitively large. Moreover a pure CNF encoding may prevent the solver from pruning the search space efficiently (Chai and Kuehlmann, 2003). Boolean satisfiability solvers available today are the result of decades of research and are deemed to be among the faster NP-hard problem specific solvers. The latest generation of SAT solvers generally have three key features (randomization of variable selection, backtracking search and some form of clause learning) and they usually run in reasonable time (even for very large instances).

A number of generalizations of SAT solvers to LPB solvers have been proposed (Pueblo (Sheini and Sakallah, 2006), Galena (Chai and Kuehlmann, 2003), OPBDP (Barth, 2005) and more). We decided to use for our tests the minisat+ LPB solver (Eén and Sörensson., 2006) because of its good results during PB evaluation 2005 (special track of the SAT ComPeTiTiOn 2005). 


\subsection{Common intervals in the Maximum Matching Model}

We propose in Figure 2 a pseudo-boolean program for computing the maximum number of common intervals between two genomes under the maximum matching model in the presence of duplications (we assume here that each gene $\mathbf{g} \in \mathcal{G}_{1} \cup \mathcal{G}_{2}$ occurs both in $G_{1}$ and in $G_{2}$; if this is not the case, a pre-process of $G_{1}$ and $G_{2}$ will delete from $G_{1}$ and $G_{2}$ those genes which appear in only one genome).

-Figure 2 should go here-

Program Common-Intervals-Matching is clearly a pseudo-boolean program, i.e., a $(0,1)$ linear program. Roughly speaking, the boolean variables are divided in two sets: true setting of variables in $C$ denote possible common intervals between $G_{1}$ and $G_{2}$, while true setting of variables in $X$ denote the mapping, i.e., matching, between $G_{1}$ and $G_{2}$.

We now turn to describing the constraints. Constraints in (C.01) and in (C.02) deal with consistency of the mapping: each gene of $G_{1}$ is mapped to at most one gene of $G_{2}$, and conversely (some genes need indeed to be deleted in case of unbalanced families).

Constraints in (C.03) ensure that each common interval is counted exactly once. The key idea here is to impose an "active border" property, i.e., if variable $c_{k, \ell}^{i, j}$ is set to 1 then genes $G_{1}[i]$ and $G_{1}[j]$ must match some distinct genes between positions $k$ and $\ell$ in $G_{2}$, and genes $G_{2}[k]$ and $G_{2}[\ell]$ must match some distinct genes between positions $i$ and $j$ in $G_{1}$. More intuitively, this constraint ensures that a common interval will not be counted more than once, by forcing each extremity of a common interval to be matched.

Constraints in (C.04) to (C.07) ensure that if $c_{k, \ell}^{i, j}=1$ then the interval $[i: j]$ of $G_{1}$ and

the interval $[k: \ell]$ of $G_{2}$ is a common interval according to the mapping induced by the 
true setting of $X$. For example, constraints in (C.04) ensure that each gene in the interval $[i: j]$ of $G_{1}$ is either not mapped or is mapped to a gene in the interval $[k: \ell]$ of $G_{2}$ (thanks to constraints in (C.01), (C.02) and (C.03), genes at position $i$ and $j$ in $G_{1}$ are actually mapped to distinct genes in $G_{2}$ if $i<j$ and $\left.c_{k, \ell}^{i, j}=1\right)$.

Finally, constraints in (C.08) force the mapping to be a maximum matching between $G_{1}$ and $G_{2}$.

Proposition 1. Program Common-Intervals-Matching correctly computes the maximum number of common intervals between $G_{1}$ and $G_{2}$ under the maximum matching model.

We briefly discuss here space issues of Program Common-Intervals-Matching. First, it is easily seen that $\# C=\Theta\left(n_{1}^{2} n_{2}^{2}\right)$ and hence that (C.03) is composed of $\Theta\left(n_{1}^{2} n_{2}^{2}\right)$ constraints. The number of constraints in (C.04) to (C.07) however does depend on the number of duplications in the two genomes. Second, $\# X=\mathcal{O}\left(n_{1} n_{2}\right)$. Clearly, the size of the set $X$ determines the number of constraints in (C.01) and (C.02) and of course strongly depends on the number of duplications in $G_{1}$ and $G_{2}$. Not surprisingly, set $X$ turns out to be of moderate size in practice. Finally, the number of constraints in (C.08) is clearly linear in the size of the two genomes. We shall soon describe (section 3.4) how to speed-up the program by reducing the number of variables and constraints. Before that, we show in the following section how to modify the Program Common-Intervals-Matching in order to compute the maximum number of common intervals between two genomes under, respectively, the exemplar matching model and the intermediate model, where at least one gene in each gene family is mapped. 


\subsection{Adapting the Program for other Models}

In this section, we point out the genericity of the pseudo-boolean framework for computing a matching that maximizes the number of common intervals between two genomes. Indeed, we show here a fast and easy way to modify the Program Common-Intervals-Matching in order to compute the maximum number of common intervals between two genomes under two other models: the exemplar matching model and the intermediate model, where at least one gene in each gene family is mapped.

We observe indeed that replacing constraints in (C.08) by a new set of constraints (C.08') - see below - in Program Common-Intervals-Matching results in the pseudo-boolean program Common-Intervals-Exemplar that computes the maximum number of common intervals between genomes $G_{1}$ and $G_{2}$ under the exemplar matching model.

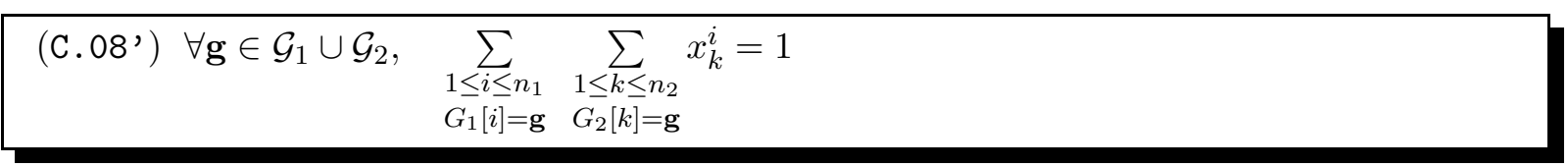

Interestingly enough, substituting now the constraints in (C.08) by a new set of constraints (C.08' ') - see below - in Program Common-Intervals-Matching results in a pseudoboolean program that computes the maximum number of common intervals between genomes $G_{1}$ and $G_{2}$ under the following intermediate model: at least one gene in each gene family is mapped. Observe that this model contains both the exemplar matching model and the maximum matching model as special cases.

$$
\left(\text { C.08' ') } \forall \mathbf{g} \in \mathcal{G}_{1} \cup \mathcal{G}_{2}, \quad \sum_{\substack{1 \leq i \leq n_{1} \\ G_{1}[i]=\mathrm{g}}} \sum_{\substack{1 \leq k \leq n_{2} \\ G_{2}[k]=\mathrm{g}}} x_{k}^{i} \geq 1\right.
$$




\subsection{Speeding-up the program}

We give in this section four rules for speeding-up Program Common-Intervals-Matching.

In theory, a very large instance may be easy to solve and a small instance hard. However, very often, small hard instances turn out be artificial, e.g., the pigeonhole problem, and hence, in case of practical instances, the running time of a pseudo-boolean solver is most of the time related to the size of the instances. The main idea here is thus to reduce the number of variables and constraints in the program (for ease of exposition, we describe our rules as filters on $C$ ). More precisely, we give rules that avoid introducing useless variables $c_{k, \ell}^{i, j}$ in $C$ in such a way that the correctness of Program Common-Intervals-Matching is maintained by repeated applications of the rules; two of these filters however do modify the correct maximum number of common intervals between the two genomes and thus ask for subsequent modifications in order to obtain the correct solution.

[Rule 1] Delete from $C$ all variables $c_{k, k}^{i, i}, 1 \leq i \leq n_{1}$ and $1 \leq k \leq n_{2}$.

Rule 1 does modify the correct number of common intervals between $G_{1}$ and $G_{2}$, and hence application of this rule asks for subsequent modifications of the number of common intervals. The key idea of Rule $\mathbf{1}$ is simply to discard common intervals of size 1 from the program. Indeed, we can compute in a pre-processing step the numbers $d_{1}$ and $d_{2}$ of genes that need to be deleted in $G_{1}$ and $G_{2}$ for obtaining a maximum matching between the two genomes. Therefore, we know that the resulting genomes will consist in $L=n_{1}-d_{1}=n_{2}-d_{2}$ genes, where

$$
L=\sum_{\mathbf{g} \in \mathcal{G}} \min \left\{\operatorname{occ}_{1}(\mathbf{g}), \operatorname{occ}_{2}(\mathbf{g})\right\}
$$


But each of these genes will contribute for 1 to the number of common intervals between $G_{1}$ and $G_{2}$, for any maximum matching. We thus simply delete all these variables and add $L$ to the number of common intervals between $G_{1}$ and $G_{2}$ found by Program Common-Intervals-Matching. [Rule 2] Delete from $C$ all variables $c_{k, \ell}^{i, j}$ for which any of the following conditions holds true:

1. $\left(\#\left\{r: k \leq r \leq \ell \wedge G_{1}[i]=G_{2}[r]\right\}=0\right) \vee\left(\#\left\{s: k \leq s \leq \ell \wedge G_{1}[j]=G_{2}[s]\right\}=0\right)$,

2. $\left(\#\left\{r: k \leq r \leq \ell \wedge G_{1}[i]=G_{2}[r]\right\}<2\right) \wedge\left(G_{1}[i]=G_{1}[j]\right)$,

3. $\left(\#\left\{p: i \leq p \leq j \wedge G_{2}[k]=G_{1}[p]\right\}=0\right) \vee\left(\#\left\{q: i \leq q \leq j \wedge G_{2}[\ell]=G_{1}[q]\right\}=0\right)$,

4. $\left(\#\left\{p: i \leq p \leq j \wedge G_{2}[k]=G_{1}[p]\right\}<2\right) \wedge\left(G_{2}[k]=G_{2}[\ell]\right)$.

Rule 2 is a quickening for constraints in (C.03). Indeed, these constraints ensure that each common interval is counted exactly once by the program by forcing the border of each common interval to be active in the computed solution, i.e., genes $G_{1}[i]$ and $G_{1}[j]$ match some distinct genes between positions $k$ and $\ell$ in $G_{2}$, and genes $G_{2}[k]$ and $G_{2}[\ell]$ match some distinct genes between positions $i$ and $j$ in $G_{1}$. Correctness of Rule 2 thus follows from the fact that Program Common-Intervals-Matching will always set a variable $c_{k, \ell}^{i, j}$ to 0 if the border property cannot be satisfied (it is assumed here that $i<j$ and $k<\ell$ ).

[Rule 3] Delete from $C$ all variables $c_{k, \ell}^{i, j}$ for which there exists at least one gene $\mathbf{g} \in \mathcal{G}$ such that $\left|\operatorname{occ}_{1}(\mathbf{g}, i, j)-\mathrm{occ}_{2}(\mathbf{g}, k, \ell)\right|>\left|\mathrm{occ}_{1}(\mathbf{g})-\mathrm{occ}_{2}(\mathbf{g})\right|$.

Roughly speaking, Rule $\mathbf{3}$ avoids us to delete too many genes in a common interval. Indeed, for one, for any $\mathbf{g} \in \mathcal{G},\left|\operatorname{occ}_{1}(\mathbf{g}, i, j)-\operatorname{occ}_{2}(\mathbf{g}, k, \ell)\right|$ is clearly the minimum number of occurrences of gene $\mathbf{g}$ that need to be deleted if $c_{k, \ell}^{i, j}=1$, i.e., $[i: j]$ and $[k: \ell]$ form a common interval between the two genomes. For another, for any $\mathbf{g} \in \mathcal{G}, \operatorname{occ}_{1}(\mathbf{g})-\mathrm{occ}_{2}(\mathbf{g}) \mid$ is the number of occurrences of gene $\mathbf{g}$ that need to be deleted in $G_{1}$ and $G_{2}$ for finding any maximum matching between the two genomes. Correctness of Rule 3 thus follows from the fact that we can certainly not delete more than $\left|\mathrm{occ}_{1}(\mathbf{g})-\mathrm{occ}_{2}(\mathbf{g})\right|$ occurrences of gene $\mathbf{g}$. 
[Rule 4] Delete from $C$ all variables $c_{k, \ell}^{i, j}$ for which the four following conditions hold true:

1. $\forall \mathrm{g} \in \mathcal{G}_{1}(i, j), \quad$ occ $_{1}(\mathrm{~g}, 1, i-1)+$ occ $_{1}\left(\mathrm{~g}, j+1, n_{1}\right)=0$,

2. $\forall \mathbf{g} \in \mathcal{G}_{2}(k, \ell), \quad \operatorname{Hocc}_{2}(\mathbf{g}, 1, k-1)+$ occ $_{2}\left(\mathbf{g}, \ell+1, n_{2}\right)=0$,

3. \#оcc $1\left(G_{1}[i]\right) \leq \operatorname{\# occ}_{2}\left(G_{1}[i]\right) \wedge$ \#occ $_{1}\left(G_{1}[j]\right) \leq$ occ $_{2}\left(G_{1}[j]\right)$,

4. \#occ $2\left(G_{2}[k]\right) \leq \# \mathrm{occ}_{1}\left(G_{2}[k]\right) \wedge$ \#оcc $_{2}\left(G_{2}[\ell]\right) \leq$ occ $_{1}\left(G_{2}[\ell]\right)$.

We first observe that Rule 4 does modify the correct number of common intervals between $G_{1}$ and $G_{2}$, and hence application of this rule asks for subsequent modifications of the number of common intervals. The rationale of Rule 4 is that, if the four conditions hold true, then $c_{k, \ell}^{i, j}$ will always be set to 1 by Program Common-Intervals-Matching. In other words, for any maximum matching between $G_{1}$ and $G_{2},[i: j]$ and $[k: \ell]$ will form a common intervals. We thus simply delete from $C$ all these variables $c_{k, \ell}^{i, j}$ and add the number of deleted variables by Rule 4 to the number of common intervals between $G_{1}$ and $G_{2}$ found by Program Common-Intervals-Matching. This rule will prove extremely useful for highly conserved regions with localized duplications.

\section{Experimental results}

As mentioned in the Introduction, the generic pseudo-boolean approach we propose in this paper can be useful for estimating the accuracy of one or several heuristic(s). In that perspective, it is necessary to compute the exact results for different datasets, that could be later used as benchmarks to which confront the results given by any given heuristic algorithm. In this section, we will compare the exact results we have obtained from a given dataset by our pseudo-boolean method to three heuristics, respectively called ILCS, IILCS and $H Y B_{2}$. 
The three heuristics we will study in the following are:

- the heuristic $I L C S$, which is a small variant of the one used in (Blin et al., 2005)

- an improvement we suggest for ILCS (that we will denote IILCS)

- a hybrid method, called $H Y B_{k}$, that mixes the approach used in the IILCS heuristic and the pseudo-boolean approach. We will mainly be interested in the case where parameter $k$ is equal to 2 .

We will thus compare the results obtained by the ILCS, IILCS and $H Y B_{2}$ heuristics to the exact results we have obtained via our pseudo-boolean approach. In particular, we will show that IILCS and $H Y B_{2}$ perform very well on the dataset we used. Before that, we first describe this dataset, and we present each of the three above mentioned heuristics in details.

\subsection{Exact Results}

We performed the computation of exact results concerning common intervals in the maximum matching model by studying the dataset used in (Blin et al., 2005). This dataset is composed of 12 complete genomes from the $13 \gamma$-Proteobacteria originally studied in (Lerat et al., 2003). The thirteenth genome (V.cholerae) was not considered, since it is composed of two chromosomes, and hence does not fit in the model we considered here for representing genomes. More precisely, this dataset is composed of the genomes of the following species:

- Buchnera aphidicola APS (Baphi, Genbank accession number NC_002528),

- Escherichia coli K12 (Ecoli, NC_000913),

- Haemophilus influenzae Rd (Haein, NC_000907), 
- Pseudomonas aeruginosa PA01 (Paeru, NC_002516),

- Pasteurella multocida Pm70 (Pmult, NC_002663),

- Salmonella typhimurium LT2 (Salty, NC_003197),

- Xanthomonas axonopodis pv. citri 306 (Xaxon, NC_003919),

- Xanthomonas campestris (Xcamp, NC_0 03902),

- Xylella fastidiosa 9a5c (Xfast, NC_002488),

- Yersinia pestis CO_92 (Ypest-C092, NC_003143),

- Yersinia pestis KIM5 P12 (Ypest-KIM, NC_004088) and

- Wigglesworthia glossinidia brevipalpis Baphi (NC_004344).

The computation of a partition of the complete set of genes into gene families, where each family is supposed to represent a group of homologous genes, is taken from (Blin et al., 2005) (this partition was actually provided to these authors by Lerat (Lerat et al., 2003)). The main characteristics (number of genes, number of gene families, percentage of duplicates) of the twelve considered genomes are summarized in Table 1.

-Table 1 should go here-

Despite the fact that the model (maximum matching model) and the measure (number of common intervals) we study are one of the most time consuming, nearly two thirds of the exact results have been computed until now. It is also interesting to note that, for three of the twelve studied genomes (namely, Buchnera aphidicola, Haemophilus influenzae and 
Wigglesworthia glossinidia brevipalpis), we have obtained all the exact results that involve those genomes.

These results, though still partial, allow us to go further ; indeed, thanks to the exact results we have obtained, we are now able to confront the results given by three different heuristics to them. Those three heuristics are now presented in details.

\subsection{The ILCS Heuristic}

We first start by describing the heuristic that we will call ILCS (Iterative Longest Common Substring). It is actually a small variant of the one used in (Blin et al., 2005). This heuristic is greedy, and works as follows:

1. Compute the Longest Common Substring (i.e., the longest contiguous word) $S$ of the two genomes, up to a complete reversal. If there are several candidates, pick one at random

2. Match all the genes of $S$ accordingly

3. Iterate the process until all possible genes have been matched (i.e., we have obtained a maximum matching)

4. Remove, in each genome, all the genes that have not been matched

5. Compute the number of common intervals that have been obtained in this solution

For instance, suppose that $G_{1}=1234567$ and $G_{2}=674516321$. Below is a brief description of the running of $I L C S$, where the parts which are underlined represent the Longest Common Substring, and where genes in bold are matched. 


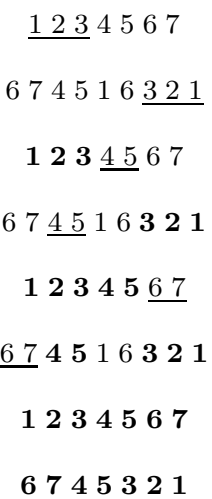

In this example, the number of Common Intervals we obtain is equal to 19.

The simple idea behind this heuristic algorithm is that a Longest Common Substring (up to complete reversal) of length $k$ contains $\frac{k(k+1)}{2}$ common intervals. Hence, finding such exact copies in both genomes intuitively helps to increase the total number of common intervals.

\subsection{The IILCS Heuristic: an improvement of $I L C S$}

A closer look on the $I L C S$ heuristic led us to suggest an improvement to it, in the form of a new heuristic, that we call IILCS (Improved Iterative Longest Common Substring). Heuristic IILCS works as follows:

1. Compute the Longest Common Substring $S$ of the two genomes, up to a complete reversal. If there are several candidates, pick one at random

2. Match all the genes of $S$ accordingly

3. Remove from genome $G_{1}$ (resp. $\left.G_{2}\right)$ the unmatched gene(s) for which there remains no unmatched genes of the same family in $G_{2}$ (resp. $\left.G_{1}\right)$

4. Iterate the process until all possible genes have been matched (i.e., we have obtained a maximum matching) 
5. Compute the number of common intervals that have been obtained in this solution

The only difference between $I I L C S$ and $I L C S$ lies in line 3: intuitively, before searching for a Longest Common Substring (up to a complete reversal), we "tidy" the two genomes. More precisely, we remove, in each genome and at each iteration, the genes for which we know they will not be matched in the final solution ; such genes are unmatched genes for which there remains no "corresponding" (i.e., of the same family) unmatched gene in the other genome. This process can simply be undertaken by counting, at each iteration, the number of unmatched genes of each gene family in both genomes. This actually allows to find Longest Common Substrings that are possibly longer than the ones found in the ILCS heuristic.

For example, let us take the same instance as the one used in the previous section, that is $G_{1}=1234567$ and $G_{2}=674516321$. Below is a brief description of the running of IILCS, where the parts which are underlined represent the Longest Common Substring, genes in italic are genes that are going to be removed (because they have no unmatched gene of the same family in the other genome), and genes in bold are matched.

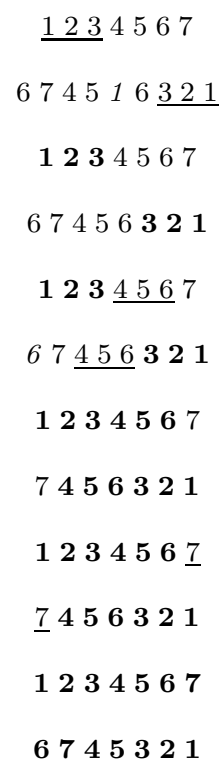


In this example, the number of Common Intervals we obtain is equal to 20.

\subsection{The Hybrid Method $H Y B_{k}$}

Starting from the IILCS heuristic, one can think of a further improvement: first, use the IILCS method until no Longest Common Substring of size greater than or equal to $k$ exists (where $k$ is a given parameter) ; this will give a partially solved instance, in the sense that some genes are matched while some are not. Second, in order to totally solve the problem, we run our pseudo-boolean program on the partially solved instance.

The $H Y B_{k}$ method can be described as follows:

1. Run the IILCS method until no Longest Common Substring of size greater than or equal to $k$ exists

2. Give the partially solved instance obtained above as an input to the pseudo-boolean program Common-Intervals-Matching

3. Compute the number of common intervals that have been obtained in this solution

Since the pseudo-boolean program will always output the best (i.e., exact) result, and since before calling the pseudo-boolean program we run the IILCS heuristic, we can ensure that $H Y B_{k}$ is at least as good as $I I L C S$ for any value of $k$ and any given instance $\left(G_{1}, G_{2}\right)$. However, the main drawback of $H Y B_{k}$ compared to IILCS concerns the running time ; indeed, IILCS is a polynomial time algorithm, while there is no guarantee that $H Y B_{k}$ (for any $k \geq 2$ ) will ever answer, even on a very powerful computer. This is because we are asking for an exact result in the second part of the algorithm, which means that it runs in exponential time. On the other hand, if we choose $k$ sufficiently small, then many genes will 
be matched by the IILCS part of the heuristic, and one can hope for the exact result to be computed relatively fast. Moreover, the exact part of the $H Y B_{k}$ heuristic is run through our pseudo-boolean program, which has been designed to speed-up the computation. In that sense, $H Y B_{k}$ appears as a compromise between the exactness of the results and the computation time.

For our experiments, we will choose the smallest possible value for $k$, that is $k=2$. The results we have obtained through our $H Y B_{k}$ method are at the same time promising and somehow disappointing ; they are promising in terms of quality of the results (more details are provided in Section 4.5), but disappointing in terms of running time. Indeed, even when fixing $k$ as small as possible (that is, $k=2$ ), only 46 out of the 66 possible pairwise comparisons were obtained within a few days of computation, that is, only 6 more results than the exact method.

\subsection{Results and Discussion}

Out of the 66 possible pairwise genome comparisons, 40 results have been obtained within a few days of computation. More precisely, among these 40 values, only 4 of them took several days to be computed (Haein/Pmult, Haein/Xfast, Paeru/Pmult and Paeru/Xfast), while the others took no more than a few minutes.

One of the main interests of our pseudo-boolean approach is that the exact results they provide can be used to test the robustness of some heuristic(s). We have thus confronted the 40 exact results we have obtained to the results given by the three heuristics we have presented in the previous sections.

First, we focus on the three genomes for which we have obtained all the exact results: results 
for Buchnera aphidicola are summarized in Table 2, results for Haemophilus influenzae are summarized in Table 3, while results concerning Wigglesworthia glossinidia brevipalpis are summarized in Table 4.

Looking at these results, we can see that, non surprisingly, $H Y B_{2}$ is always better or as good as $I I L C S$, which is itself always better or as good as $I L C S$. In particular, $H Y B_{2}$ returns the optimal value in all the cases for Buchnera aphidicola, in 5 out of the 11 cases for Haemophilus influenzae, and in 9 out of the 11 cases for Wigglesworthia glossinidia brevipalpis.

-Table 2 should go here-

-Table 3 should go here-

-Table 4 should go here-

A more global view at the respective performances of heuristics $I L C S, I I L C S$ and $\mathrm{HYB}_{2}$ is given in Table 5. In this table, for each of the 40 exact results we have obtained, and for each heuristic $\mathcal{H}$, we provide the ratio $\frac{I C(\mathcal{H})}{I C(O P T)}$, where $I C(\mathcal{H})$ is the number of common intervals returned by $\mathcal{H}$ and $I C(O P T)$ is the exact number of common intervals. This ratio is given as a percentage. These results are also illustrated in a graphical form in Figure 3.

These results confirm that $H Y B_{2}$ is always better (or as good as) IILCS, which is always better (or as good as) ILCS: more precisely, on average, IILCS improves by $2.66 \%$ the performances of $I L C S$, while $H Y B_{2}$ improves by a further $0.98 \%$ the performances of $I I L C S$.

What is even more striking is that both heuristics IILCS and $H Y B_{2}$ appear to be very 
good on the dataset we studied. Indeed, out of the 40 instances for which we have computed the exact results, IILCS returns the optimal result in 7 cases, and returns a number of common intervals that is more than $99 \%$ of the optimal number for 22 other cases. The "worse" result that IILCS provides is $93.18 \%$ away from the optimal (Xfast/Ypest-CO92). On average, over the 40 pairwise comparisons for which we have exact results, IILCS gives a number of common intervals that is $98.9 \%$ of the optimal number.

Concerning heuristic $H Y B_{2}$, out of the 40 instances for which we have computed the exact results, it returns the optimal result in 23 cases, and returns a number of common intervals that is more than $99 \%$ of the optimal number for the remaining 17 other cases. The "worse" result that $\mathrm{HYB}_{2}$ provides is $99.22 \%$ away from the optimal (Pmult/Ypest-KIM). On average, over the 40 pairwise comparisons for which we have exact results, $H Y B_{2}$ gives a number of common intervals that is $99.88 \%$ of the optimal number.

-Table 5 should go here-

-Figure 3 should go here-

These results come as a surprise, because, despite being extremely simple and fast, IILCS appears to be extremely good on this dataset. $H Y B_{2}$ works even better on this dataset, but its main drawback is that we have no guarantee on its execution time. Hence, this strongly suggests that computing common intervals in the maximum matching model can simply be undertaken using either IILCS or $H Y B_{2}$ (depending on the execution time of $H Y B_{2}$ ), while remaining accurate, thus validating these heuristics. 


\section{Conclusion}

In this paper, we have introduced a novel and original method that helps speeding-up computations of exact results for comparing whole genomes containing duplicates. This method makes use of pseudo-boolean programming. Our approach is very general, and can handle several (dis)similarity measures (breakpoints, common intervals, conserved intervals, MAD, etc.) under several possible models (exemplar matching model, maximum matching model, but also most models within those two extrema).

An example of such an approach (common intervals under the maximum matching model) has been developed, in order to illustrate the main ideas of the pseudo-boolean transformation framework that we suggest. Experiments have also been undertaken on a dataset of $\gamma$-Proteobacteria, showing the validity of our approach, since 40 results (out of 66) have been

obtained in a limited amount of time. Moreover, these preliminary results have allowed us to state that, in particular, both the IILCS and the $H Y B_{2}$ heuristics provide excellent results on this dataset, hence showing their validity and robustness (though there is no guarantee on the execution time of $H Y B_{2}$ ). On the whole, these preliminary results are very encouraging.

Our approach is in fact more ambitious. Our long term goal is indeed to develop a generic pseudo-boolean approach for the exact computation of various genome distances (number of breakpoints, number of common intervals, number of conserved intervals, MAD, etc.) under both the exemplar and the maximum matching models, and use this generic approach on different datasets. The rationale of this approach is threefold:

1. There is a crucial need for new algorithmic solutions providing exact genome distances under both the exemplar and the maximum matching models in order to estimate 
the accuracy of existing heuristics and to design new efficient biologically relevant heuristics.

2. Very little is known about the relations between the various genome distances that have been defined so far (number of breakpoints, number of common intervals, number of conserved intervals, MAD, etc.). We thus propose to extensively compare all these genome distances under both models with a generic pseudo-boolean framework on several datasets.

3. We also plan to further investigate the relations between the exemplar and the maximum matching models. We strongly believe here that, in the light of these comparisons, some biologically relevant intermediate model between these two extrema could be defined.

There is still a great amount of work to be done, and some of it is being undertaken by the authors at the moment. Among other things, one can cite:

- Implementing and testing all the possible above mentioned models, for all the possible above mentioned (dis)similarity measures,

- For each case, determining strong and relevant rules for speeding-up the process by avoiding the generation of a large number clauses and variables (a pre-processing step that should not be underestimated),

- Obtaining exact results for each of these models and measures, and for different datasets, that could be later used as benchmarks in order to validate (or not) possible heuristics. 


\section{References}

Barth, P. (2005). A Davis-Putnam based enumeration algorithm for linear pseudo-Boolean optimization. Technical Report MPI-I-95-2-003, Max Planck Institut Informatik. 13 pages.

Bergeron, A., Chauve, C., de Montgolfier, F., and Raffinot, M. (2005). Computing common intervals of $\mathrm{K}$ permutations, with applications to modular decomposition of graphs. In Proc. 5th Workshop on Algorithms in Bioinformatics (WABI), volume 3669 of LNCS, pages $779-790$.

Blin, G., Chauve, C., and Fertin, G. (2004). The breakpoint distance for signed sequences. In Proc. 1st Algorithms and Computational Methods for Biochemical and Evolutionary Networks (CompBioNets), pages 3-16. KCL publications.

Blin, G., Chauve, C., and Fertin, G. (2005). Genes order and phylogenetic reconstruction: Application to $\gamma$-proteobacteria. In Proc. 3rd RECOMB Comparative Genomics Satellite Workshop, volume 3678 of $L N B I$, pages 11-20.

Blin, G. and Rizzi, R. (2005). Conserved intervals distance computation between nontrivial genomes. In Proc. 11th Annual Int. Conference on Computing and Combinatorics (COCOON), volume 3595 of $L N C S$, pages 22-31.

Bourque, G., Yacef, Y., and El-Mabrouk, N. (2005). Maximizing synteny blocks to identify ancestral homologs. In Proc. 3rd RECOMB Comparative Genomics Satellite Workshop, volume 3678 of $L N B I$, pages $21-35$.

Bryant, D. (2000). The complexity of calculating exemplar distances. In Comparative Genomics: Empirical and Analytical Approaches to Gene Order Dynamics, Map Align- 
ment, and the Evolution of Gene Families, pages 207-212. Kluwer.

Chai, D. and Kuehlmann, A. (2003). A fast pseudo-boolean constraint solver. In Proc. 40th ACM IEEE conference on Design automation, pages 830-835.

Chauve, C., Fertin, G., Rizzi, R., and Vialette, S. (2006). Genomes containing duplicates are hard to compare. In Proc Int. Workshop on Bioinformatics Research and Applications (IWBRA), volume 3992 of LNCS, pages 783-790.

Chen, X., Zheng, J., Fu, Z., Nan, P., Zhong, Y., Lonardi, S., and Jiang, T. (2005). Assignment of orthologous genes via genome rearrangement. IEEE/ACM Transactions on Computational Biology and Bioinformatics, 2(4):302-315.

Chen, Z., Fowler, R., Fu, B., and Zhu, B. (2006a). Lower bounds on the approximation of the exemplar conserved interval distance problem of genomes. In Proc. 12th Annual International Computing and Combinatorics Conference (COCOON'06), volume 4112 of $L N C S$, pages $245-254$.

Chen, Z., Fu, B., and Zhu, B. (2006b). The approximability of the exemplar breakpoint distance problem. In Proc. 2nd International Conference on Algorithmic Aspects in Information and Management (AAIM'06), volume 3328 of LNCS, pages 291-302.

Chrobak, M., Kolman, P., and Sgall, J. (2005). The greedy algorithm for the minimum common string partition problem. ACM Transactions on Algorithms, 1(2):350-366.

Eén, N. and Sörensson., N. (2006). Translating pseudo-boolean constraints into SAT. Journal on Satisfiability, Boolean Modeling and Computation, 2:1-26.

El-Mabrouk, N. (2001). Sorting signed permutations by reversals and insertions/deletions of contiguous segments. Journal of Discrete Algorithms, 1(1):105-122. 
El-Mabrouk, N. (2002). Reconstructing an ancestral genome using minimum segments duplications and reversals. Journal of Computer and System Sciences, 65:442-464.

Fu, Z., Chen, X., Vacic, V., Nan, P., Zhong, Y., and Jiang, T. (2006). A parsimony approach to genome-wide ortholog assignment. In Proc. 10th Annual International Conference on Research in Computational Molecular Biology (RECOMB), volume 3909 of $L N B I$, pages $578-594$.

Goldstein, A., Kolman, P., and Zheng, J. (2004). Minimum common string partition problem: Hardness and approximations. In Proc. 15th International Symposium on Algorithms and Computation (ISAAC), volume 3341 of LNCS, pages 484-495.

Kolman, P. and Waleń, T. (2006). Reversal distance for strings with duplicates: Linear time approximation using hitting set. In Proc. 4th Workshop on Approximation and Online Algorithms (WAOA), volume 4368 of LNCS, pages 281-291.

Kolman, P. and Waleń, T. (2007). Approximating reversal distance for strings with bounded number of duplicates. Discrete Applied Mathematics, 155(3):327-336.

Landau, G., Parida, L., and Weimann, O. (2005). Gene proximity analysis across whole genomes via PQ trees. Journal of Computational Biology, 12(10):1289-1306.

Lerat, E., Daubin, V., and Moran, N. (2003). From gene tree to organismal phylogeny in prokaryotes: the case of $\gamma$-proteobacteria. PLoS Biology, 1(1):101-109.

Marron, M., Swenson, K., and Moret, B. (2004). Genomic distances under deletions and insertions. Theoretical Computer Science, 325(3):347-360.

Sankoff, D. (1999). Genome rearrangement with gene families. Bioinformatics, 15(11):909917. 
Sankoff, D. and Haque, L. (2005). Power boosts for cluster tests. In Proc. 3rd RECOMB Comparative Genomics Satellite Workshop, volume 3678 of LNBI, pages 11-20.

Schrijver, A. (1998). Theory of Linear and Integer Programming. John Wiley and Sons.

Sheini, H. and Sakallah, K. (2006). Pueblo: A hybrid pseudo-boolean SAT solver. Journal on Satisfiability, Boolean Modeling and Computation, 2:165-189.

Swenson, K., Marron, M., Earnest-DeYoung, J., and Moret, B. (2005a). Approximating the true evolutionary distance between two genomes. In Proc. 7th Workshop on Algorithms Engineering and Experiments and Second Workshop on Analytic Algorithmics and Combinatorics (ALENEX, SIAM Press, pages 121-129.

Swenson, K., Pattengale, N., and Moret, B. (2005b). A framework for orthology assignment from gene rearrangement data. In Proc. 3rd RECOMB Workshop on Comparative Genomics (RECOMB-CG), volume 3678 of $L N B I$, pages $153-166$.

Thach, N. C. (2005). Algorithms for calculating exemplar distances. Honours Year Project Report, National University of Singapore.

Uno, T. and Yagiura, M. (2000). Fast algorithms to enumerate all common intervals of two permutations. Algorithmica, 26(2):290-309. 


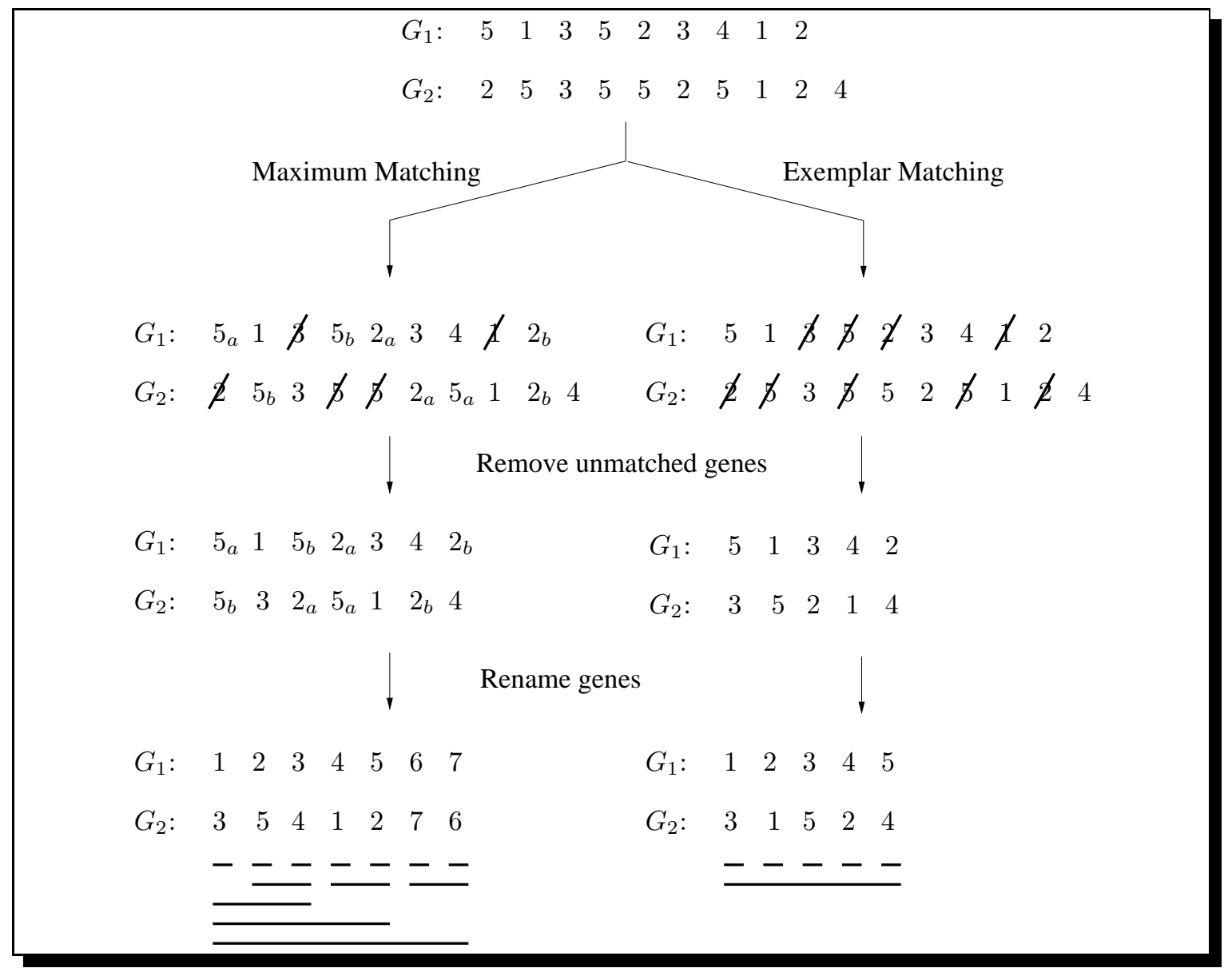

Figure 1: Illustration of the maximum matching and the exemplar matching concepts. Common intervals, in each of the two cases, are shown with bold lines in the bottom part of the figure 


\section{Program Common-Intervals-Matching}

\section{objective:}

$$
\operatorname{maximize} \sum_{c_{k, \ell}^{i, j} \in C} c_{k, \ell}^{i, j}
$$

variables:

$$
\begin{aligned}
& C=\left\{c_{k, \ell}^{i, j}: 1 \leq i \leq j \leq n_{1} \wedge 1 \leq k \leq \ell \leq n_{2}\right\} \\
& X=\left\{x_{k}^{i}: 1 \leq i \leq n_{1} \wedge 1 \leq k \leq n_{2} \wedge G_{1}[i]=G_{2}[k]\right\}
\end{aligned}
$$

\section{subject to:}

$$
\begin{aligned}
& \text { (C.01) } \forall i=1,2, \ldots, n_{1}, \quad \sum_{1 \leq k \leq n_{2}} x_{k}^{i} \leq 1 \\
& \text { (C. 02) } \forall k=1,2, \ldots, n_{2}, \quad \sum_{1 \leq i \leq n_{1}} x_{k}^{i} \leq 1 \\
& G_{1}[i]=G_{2}[k] \\
& \text { (C.03) } \forall c_{k, \ell}^{i, j} \in C, \quad 4 c_{k, \ell}^{i, j}-\sum_{k \leq r \leq \ell} x_{r}^{i}-\sum_{k \leq s \leq \ell} x_{s}^{j}-\sum_{i \leq p \leq j} x_{k}^{p}-\sum_{i \leq q \leq j} x_{\ell}^{q} \leq 0 \\
& G_{1}[i]=G_{2}[r] \quad G_{1}[j]=G_{2}[s] \quad G_{1}[p]=G_{2}[k] \quad G_{1}[q]=G_{2}[\ell]
\end{aligned}
$$

\section{domains:}

$$
\begin{aligned}
& \forall x_{k}^{i} \in X, \quad x_{k}^{i} \in\{0,1\} \\
& \forall c_{k, \ell}^{i, j} \in C, \quad c_{k, \ell}^{i, j} \in\{0,1\}
\end{aligned}
$$

Figure 2: Program Common-Intervals-Matching for finding the maximum number of common intervals between two genomes under the maximum matching model 


\begin{tabular}{|c||c|c|c|}
\hline \multicolumn{4}{|c|}{ Main characteristics } \\
\hline \hline Genome & Number & Number of & Percentage \\
of genes & gene families & of duplications \\
\hline \hline Baphi & 564 & 549 & 2.66 \\
\hline Ecoli & 4183 & 3423 & 18.17 \\
\hline Haein & 1709 & 1531 & 10.42 \\
\hline Paeru & 5540 & 4500 & 18.77 \\
\hline Pmult & 2015 & 1811 & 10.12 \\
\hline Salty & 4203 & 3456 & 17.77 \\
\hline Wglos & 653 & 627 & 3.98 \\
\hline Xaxon & 4192 & 3634 & 13.31 \\
\hline Xcamp & 4029 & 3468 & 13.92 \\
\hline Xfast & 2680 & 2346 & 12.46 \\
\hline Ypest-CO92 & 3599 & 3021 & 16.06 \\
\hline Ypest-KIM & 3879 & 3236 & 16.58 \\
\hline
\end{tabular}

Table 1: Main characteristics of the twelve considered $\gamma$-Proteobacteria genomes

\begin{tabular}{|c|c|c|c|c|}
\hline \multicolumn{5}{|c|}{ Baphi vs } \\
\hline & $I L C S$ & $I I L C S$ & $H Y B_{2}$ & Exact \\
\hline Ecoli & 2605 & 2869 & 2882 & 2882 \\
\hline Haein & 1103 & 1109 & 1109 & 1109 \\
\hline Paeru & 1492 & 1518 & 1524 & 1524 \\
\hline Pmult & 1216 & 1224 & 1224 & 1224 \\
\hline Salty & 2641 & 2849 & 2849 & 2849 \\
\hline Wglos & 1261 & 1267 & 1275 & 1275 \\
\hline Xaxon & 1127 & 1183 & 1183 & 1183 \\
\hline Xcamp & 1147 & 1183 & 1183 & 1183 \\
\hline Xfast & 975 & 979 & 979 & 979 \\
\hline Ypest-CO92 & 2387 & 2541 & 2585 & 2585 \\
\hline Ypest-KIM & 1965 & 2124 & 2141 & 2141 \\
\hline
\end{tabular}

Table 2: Comparison of the results - Buchnera aphidicola 


\begin{tabular}{|c|c|c|c|c|}
\hline \multicolumn{5}{|c|}{ Haein vs } \\
\hline & $I L C S$ & $I I L C S$ & $H Y B_{2}$ & Exact \\
\hline Baphi & 1103 & 1109 & 1109 & 1109 \\
\hline Ecoli & 2758 & 2774 & 2783 & 2784 \\
\hline Paeru & 1980 & 2016 & 2026 & 2036 \\
\hline Pmult & 3901 & 3910 & 3911 & 3936 \\
\hline Salty & 2794 & 2809 & 2819 & 2820 \\
\hline Wglos & 1077 & 1085 & 1085 & 1085 \\
\hline Xaxon & 1446 & 1468 & 1469 & 1471 \\
\hline Xcamp & 1441 & 1455 & 1458 & 1458 \\
\hline Xfast & 1285 & 1293 & 1295 & 1295 \\
\hline Ypest-CO92 & 2668 & 2686 & 2694 & 2694 \\
\hline Ypest-KIM & 2458 & 2487 & 2499 & 2500 \\
\hline
\end{tabular}

Table 3: Comparison of the results - Haemophilus influenzae

\begin{tabular}{|c|c|c|c|c|}
\hline \multicolumn{5}{|c|}{ Wglos vs } \\
\hline & $I L C S$ & $I I L C S$ & $H Y B_{2}$ & Exact \\
\hline Baphi & 1261 & 1267 & 1275 & 1275 \\
\hline Ecoli & 2102 & 2184 & 2328 & 2328 \\
\hline Haein & 1077 & 1085 & 1085 & 1085 \\
\hline Paeru & 1496 & 1506 & 1552 & 1558 \\
\hline Pmult & 1204 & 1211 & 1214 & 1214 \\
\hline Salty & 2083 & 2274 & 2331 & 2335 \\
\hline Xaxon & 1120 & 1198 & 1225 & 1225 \\
\hline Xcamp & 1151 & 1196 & 1223 & 1223 \\
\hline Xfast & 963 & 973 & 994 & 994 \\
\hline Ypest-CO92 & 2037 & 2301 & 2318 & 2318 \\
\hline Ypest-KIM & 1833 & 2086 & 2093 & 2093 \\
\hline
\end{tabular}

Table 4: Comparison of the results - Wigglesworthia glossinidia brevipalpis 


\begin{tabular}{|c|c|c|c|c|c|c|c|c|c|}
\hline Genome 1 & Genome 2 & ILCS & IILCS & HYB2 & Genome 1 & Genome 2 & ILCS & IILCS & HYB2 \\
\hline Baphi & Ecoli & 90.39 & 99.55 & 100 & Haein & Xcamp & 98.83 & 99.79 & 100 \\
\hline Baphi & Haein & 99.46 & 100 & 100 & Haein & Xfast & 99.23 & 99.85 & 100 \\
\hline Baphi & Paeru & 97.9 & 99.61 & 100 & Haein & Ypest-CO92 & 99.03 & 99.7 & 100 \\
\hline Baphi & Pmult & 99.35 & 100 & 100 & Haein & Ypest-KIM & 98.32 & 99.48 & 99.96 \\
\hline Baphi & Salty & 92.7 & 100 & 100 & Paeru & Pmult & 97.48 & 99.32 & 99.74 \\
\hline Baphi & Wglos & 98.9 & 99.37 & 100 & Paeru & Wglos & 96.02 & 96.66 & 99.61 \\
\hline Baphi & Xaxon & 95.27 & 100 & 100 & Paeru & Xfast & 98.11 & 99.5 & 99.71 \\
\hline Baphi & Xcamp & 96.96 & 100 & 100 & Pmult & Salty & 98.16 & 99.23 & 99.56 \\
\hline Baphi & Xfast & 99.59 & 100 & 100 & Pmult & Wglos & 99.18 & 99.75 & 100 \\
\hline Baphi & Ypest-CO92 & 92.34 & 98.3 & 100 & Pmult & Ypest-CO92 & 98.15 & 99.03 & 99.42 \\
\hline Baphi & Ypest-KIM & 91.78 & 99.21 & 100 & Pmult & Ypest-KIM & 97.83 & 98.19 & 99.22 \\
\hline Ecoli & Haein & 99.07 & 99.64 & 99.96 & Salty & Wglos & 89.21 & 97.39 & 99.83 \\
\hline Ecoli & Pmult & 98.65 & 99.61 & 99.97 & Salty & Xfast & 98.74 & 99.44 & 99.9 \\
\hline Ecoli & Wglos & 90.29 & 93.81 & 100 & Wglos & Xaxon & 91.43 & 97.8 & 100 \\
\hline Ecoli & Xfast & 97.39 & 98.4 & 99.79 & Wglos & Xcamp & 94.11 & 97.79 & 100 \\
\hline Haein & Paeru & 97.25 & 99.02 & 99.51 & Wglos & Xfast & 96.88 & 97.89 & 100 \\
\hline Haein & Pmult & 99.11 & 99.34 & 99.36 & Wglos & Ypest-CO92 & 87.88 & 99.27 & 100 \\
\hline Haein & Salty & 99.08 & 99.61 & 99.96 & Wglos & Ypest-KIM & 87.58 & 99.67 & 100 \\
\hline Haein & Wglos & 99.26 & 100 & 100 & Xfast & Ypest-CO92 & 92.2 & 93.18 & 100 \\
\hline Haein & Xaxon & 98.3 & 99.8 & 99.86 & Xfast & Ypest-KIM & 98.04 & 98.31 & 99.89 \\
\hline \multicolumn{4}{|c|}{ Heuristic } & \multicolumn{2}{|c|}{ ILCS } & \multicolumn{2}{|c|}{ IILCS } & \multicolumn{2}{|c|}{ НYB2 } \\
\hline \multicolumn{4}{|c|}{ Average performance } & \multicolumn{2}{|c|}{96.24} & \multicolumn{2}{|l|}{98.9} & \multicolumn{2}{|c|}{99.88} \\
\hline
\end{tabular}

Table 5: Performances of each of the three heuristics compared to the exact result (40 instances) 


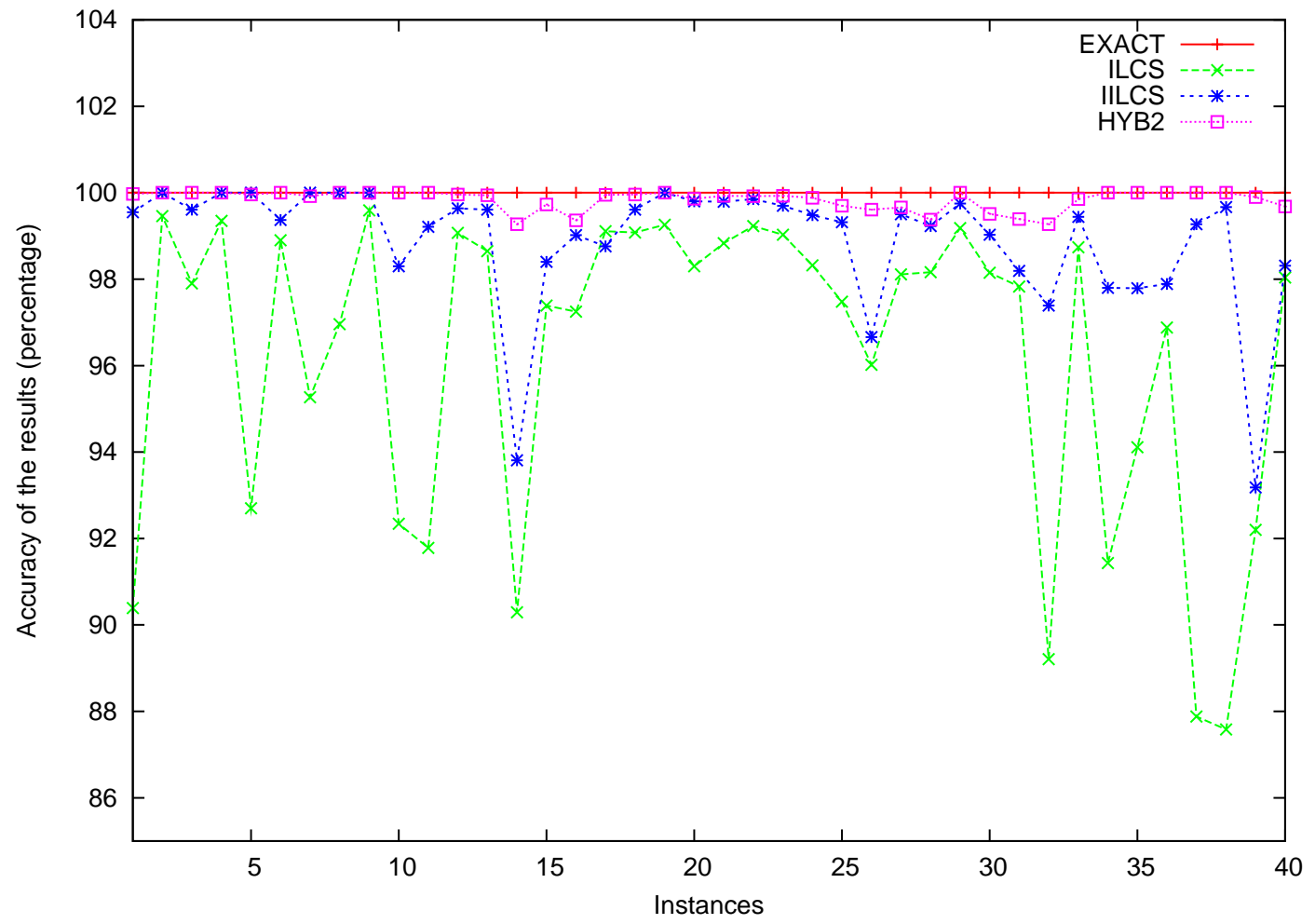

Figure 3: Graphical comparison of the exact results (100\%) with the three heuristics ILCS, IILCS and $\mathrm{HYB}_{2}$ 